\title{
Pain levels and typical symptoms of acute endodontic infections: a prospective, observational study
}

\author{
Dan-Krister Rechenberg ${ }^{1 *}$, Ulrike Held ${ }^{2}$, Jakob M. Burgstaller ${ }^{2}$, Gabriel Bosch and Thomas Attin ${ }^{1}$
}

\begin{abstract}
Background: This study aimed to identify key symptoms that could be associated with the diagnosis of acute forms of symptomatic apical periodontitis (SAP) and symptomatic irreversible pulpitis (SIP), and to identify a diagnostic algorithm based on these symptoms.

Methods: In this prospective, observational study 173 emergency patients diagnosed with acute pain of endodontic origin and no swelling or fistula were included. Patients were asked 11 specific questions from a checklist with a possible discerning value between acute SAP and acute SIP. Pain levels were recorded using the numeric rating scale (NRS-11). Subsequently, the painful tooth was diagnosed. Logistic regression was used to evaluate the checklist regarding its differentiation between SAP $(N=103)$ and SIP $(N=70)$. Moreover, a decision tree was constructed based on recursive partitioning to identify a hierarchy in differentiating symptoms.

Results: With identical median NRS-11 scores of 8, the teeth diagnosed with acute SAP and SIP were severely painful. The decision tree analysis resulted in a tree with splits according to pain on cold, perceived tooth extrusion, and pain duration. The overall sensitivity of the tree to detect SAP based on key symptoms was $95 \%$, its specificity was $31 \%$.

Conclusions: The best indicator for SAP was a reported absence of pain to cold stimuli. In teeth that did have a history of pain triggered by cold stimuli, the decision tree correctly identified SAP in $72 \%$ of the teeth that felt too high and had hurt for less than one week.
\end{abstract}

Keywords: Pain, Root canal, Symptom, Diagnostic

\section{Background}

Odontalgia is the main cause of oro-facial pain [1]. Acute dental pain is mostly attributed to microbial infection of the dentin being in close proximity to the pulp, the pulp space and finally the periapical tissues $[2,3]$. The pulp, the periodontal ligament, and the periapical tissues form natural barriers, which help the host orchestrate a defense against invading opportunistic pathogens [4]. When these barriers are invaded with pathogens nociceptors are activated due to inflammation and tissue breakdown [5]. Three symptomatic clinical conditions deriving from endodontically involved teeth

\footnotetext{
* Correspondence: dan.rechenberg@zzm.uzh.ch

${ }^{1}$ Department of Preventive Dentistry, Periodontology and Cariology, Center of Dental Medicine, University of Zürich, Switzerland, Plattenstrasse 11, CH-8032 Zürich, Switzerland

Full list of author information is available at the end of the article
}

have been identified: symptomatic irreversible pulpitis (SIP), symptomatic apical periodontitis (SAP) and acute apical abscess (AAA) [6]. Even though these can be extremely painful $[2,7]$, they do not need to be $[8,9]$. Unfortunately, the current diagnostic nomenclature of the American Association of Endodontists (AAE; [6]) does not differentiate between teeth that cause significant enough pain to require the patient to seek emergency care from those, which merely show an increased reaction to diagnostic tests [10].

The socioeconomic importance of dental pain has long been recognized [11]. It has thus been attempted to develop specific dental pain questionnaires for epidemiologic studies [12]. These questionnaires appear to have good predictive values to differentiate between groups of conditions, such as those that are caused by endodontic 
infections and those that are not. However, they do not differentiate the main causes of severe dental pain emanating from endodontic origin. There have been other approaches, which were more specific. However, these frequently mixed symptoms reported by the patients with clinical observations by the investigators [13, 14]. The significance of establishing the correct diagnosis of endodontic infections should not be under-estimated [15]. If endodontic infections are not treated appropriately, life-threatening conditions can evolve [16]. Due to the obvious clinical symptom of edema (swelling) associated with the diagnosis of AAA, this diagnosis poses no challenge. In contrast, clear-cut symptoms have not been identified to differentiate between SAP and SIP. While SIP is merely painful, SAP is the beginning of the spread of infection with a possibility of untoward systemic consequences [16]. Abscess formation, where bacteria are invariably present in the periodical tissues, marks one possible endpoint of this infection process [17]. Depending on the severity of the infection and the location of the tooth, SAP can even lead to the death of the patient if not treated appropriately [18]. Furthermore, the emergency treatment for SIP and SAP differs [15]. With SIP, simply removing the coronal pulp is sufficient for relief [19], while with SAP the disinfection of the entire root canal system is needed. It would thus be helpful to further investigate symptoms including pain levels and pain duration that can be specifically related to the acute forms of SIP and SAP.

In this prospective, observational study, adult patients seeking emergency care in a dental hospital because of inflammatory conditions caused by infection of the pulp space were assessed. The aim of the study was to identify key symptoms that could be associated with either SIP or SAP, and to identify a diagnostic algorithm based on these symptoms. Symptoms were related to clinical signs/findings.

\section{Methods}

\section{Cohort identification and inclusion criteria}

All patients attending the dental emergency unit at our institution from opening at 07:30 am to 10 am were considered. It was aimed to include all adult (18 years or older) patients presenting with acute pain from a permanent tooth caused by an endodontic infection. The emergency unit consisted of physicians and dentists from all dental specialties. After a short first examination by an oral surgeon, patients diagnosed with pericoronitis or temporomandibular joint pain were referred to the Oral Surgery department. All other patients were referred to the Department of Preventive Dentistry, Periodontology and Cariology for further examination. Patients who did not present with spontaneous pain, but merely reported slight discomfort to stimuli indicative of reversible pulpitis were not considered as'acute'. These patients did not enter the study. The remaining patients presenting with acute pain from a permanent tooth were asked to participate in the study (Fig. 1). Written informed consent was obtained from all patients. The current study protocol was approved by the local ethics committee (KEK-ZH-Nr. 2012-0450) and was conducted in accordance with the Declaration of the World Medical Association. Moreover, it was confirmed that this investigation conformed to STROBE guidelines for observational studies. Patients were excluded from the study if they: (i) were not able to clearly communicate in German or English language, (ii) refused to participate, (iii) refused to be, or could not be (pregnancy) diagnosed radiographically, (iv) were on immunosuppressant or long-term anti-inflammatory medication, or took antibiotics during the last 3 weeks, (v) had already initiated treatment of their pain-causing tooth/condition, (vi) could not be diagnosed clearly, (vii) had a condition that was not due to an endodontic infection, or (viii) were diagnosed with an acute apical abscess.

\section{Checklist for key symptoms and pain assessment}

Participants who fulfilled the inclusion criteria were interviewed using a checklist with 11 dichotomous questions with a possible discerning value between SIP and SAP (Table 1). This checklist listed symptoms that have been reported in the endodontic literature [20]. It also contained an assessment of pain intensity and duration. The checklist was originally written in German and translated to English for international patients. It was piloted by the authors of this communication and later filled in by either one of two investigators (DKR and GB). Patients were guided through these questions by one of these two investigators. Patients who could not communicate clearly were excluded. In addition, the pain intensity of the presenting condition was assessed using the numeric rating scale (NRS-11; [21]). The examiner asked the patient to quantify his/her maximum pain intensity within the last $24 \mathrm{~h}$ on a scale from 0 to 10 . The following anchors were used to describe the rating scale: ${ }^{\prime} 0^{\prime}=$ no pain/pain free and $' 10$ ' = worst pain imaginable.

\section{Diagnosis}

The clinical evaluation included cold testing with carbon dioxide snow, assessment of tenderness to percussion, tooth mobility, and periodontal probing depths. Moreover, the soft tissues were checked for tenderness to palpation, signs of erythema, and presence of a sinus tract or swelling. The findings were compared to a healthy, contralateral tooth that served as a control. Radiographic examination was performed using single-tooth 
Number of patients:

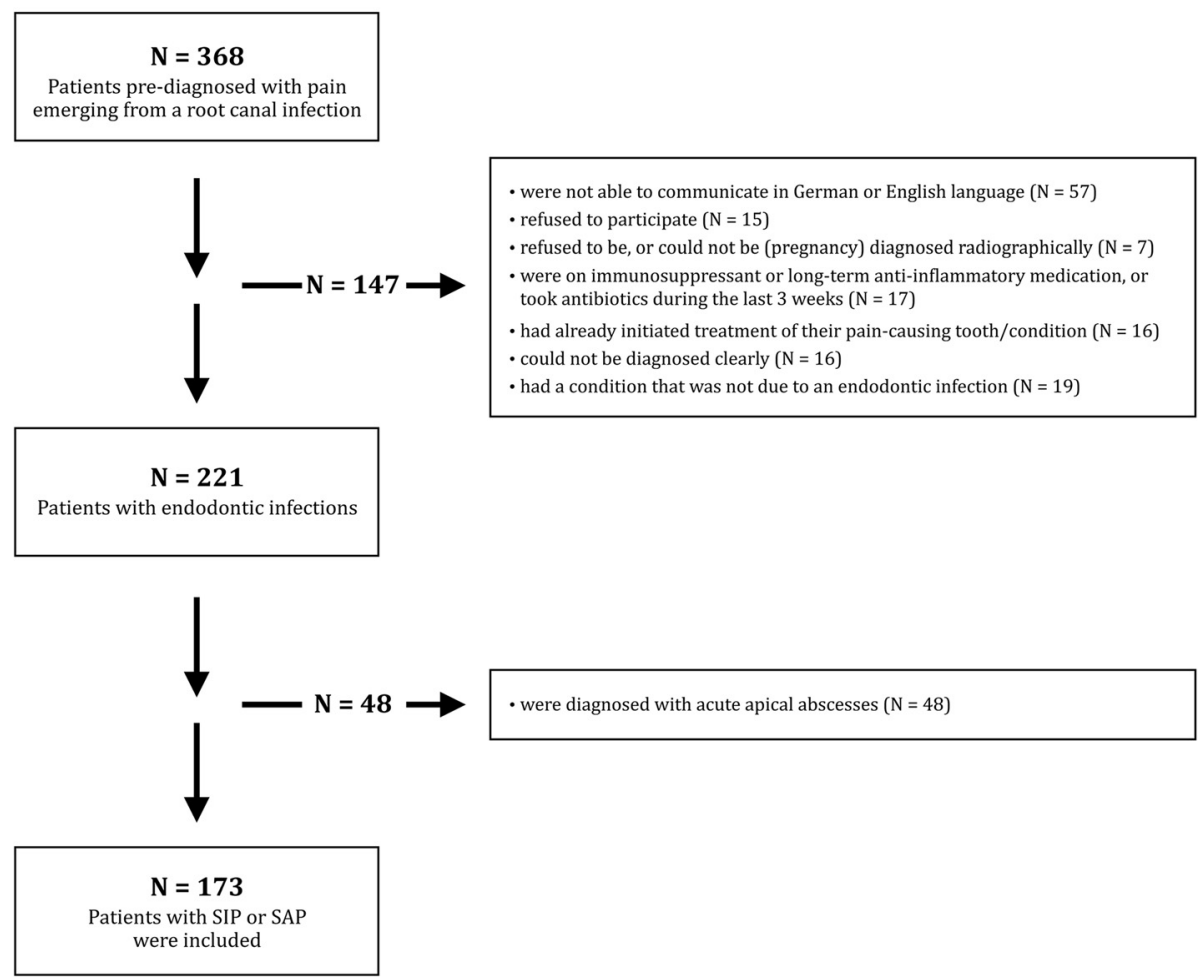

Fig. 1 Flow chart depicting the decisions why patients were excluded from the study. All emergency patients presenting with severe pain originating from a single endodontically involved tooth in the course of 64 weeks were initially considered. Teeth diagnosed with an acute apical abscess were then excluded. SIP symptomatic irreversible pulpitis, SAP symptomatic apical periodontitis.

radiographs (Digora, Soredex, Tuusula, Finland). The different inflammatory endodontic conditions (SIP, SAP, or AAA) were established according to the recommended diagnostic terminology of the Consensus Conference of the AAE [6]. In a deviation from that nomenclature, however, each tooth was assigned only one main diagnosis (Table 2). The examiner noted this diagnosis, together with relevant data (date, gender, age, analgesics taken in the past $24 \mathrm{~h}$ ) in an anonymized data sheet.

\section{Statistics and data evaluation}

Descriptive statistics included counts and percentages for the questions on pain history. Categorical data was compared between groups using the Chi-squared test. NRS-11 pain levels, which are non-interval ratings, were compared between groups using Mann-Whitney $U$ test. The alpha-type error was set at $5 \%$. To assess the diagnostic value of the 11 symptoms (other than pain) between SAP and SIP, a multiple logistic regression model was fitted to the outcome variable SAP. All of the variables of the checklist were included in order to obtain a predicted probability for SAP for each individual patient. Because some teeth were bridge abutments and the question did thus not make sense, the variable "Tooth feels too high" had 11 missing values. These were multiply imputed with 5 replications. Results of the logistic regression are based on the pooled estimates of the 5 imputed data sets. The area under the receiver operating characteristic (AUC) curve was used to evaluate the discriminative ability of the regression model.

In an alternative approach recursive partitioning was used to construct a decision tree. The focus of the tree was to facilitate the diagnostic decision between SAP and SIP. All variables of the checklist were included in the tree model. All analyses were conducted using $\mathrm{R}$ statistical software [22].

\section{Results}

\section{Study population and teeth}

From January 2013 over a period of 15 month (64 weeks) 368 adult patients attended the dental emergency unit at our institution with severe pain on a permanent tooth. One hundred and forty-seven were not eligible to enter the study because they did not meet the criteria for inclusion (Fig. 1). From the 221 patients with acute pain of endodontic origin, 70 were diagnosed with SIP, 103 with SAP and 48 with AAA. The 48 individuals diagnosed with AAA were also excluded from analysis (Fig. 1). Of 
Table 1 History checklist for dental emergency patients asking for symptoms to possibly discern between SIP and SAP

\begin{tabular}{|c|c|c|}
\hline$\#$ & Question & Answer options \\
\hline 1 & Has the pain interfered with sleep? & $y / n$ \\
\hline 2 & $\begin{array}{l}\text { Has the pain ever been stronger than during the } \\
\text { last } 24 \mathrm{~h} \text { ? }\end{array}$ & $y / n$ \\
\hline 3 & Did the pain start less than 1 week ago? & $y / n$ \\
\hline 4 & Has the pain been sporadic or constant? & $\begin{array}{l}\text { sporadic/ } \\
\text { constant }\end{array}$ \\
\hline 5 & Has the pain been localized or radiating? & $\begin{array}{l}\text { localized/ } \\
\text { radiating }\end{array}$ \\
\hline 6 & How was the main quality of the pain? & dull/sharp \\
\hline 7 & Has chewing increased the pain? & $y / n$ \\
\hline 8 & Have warm drinks/food increased the pain? & $y / n$ \\
\hline 9 & Has cold increased the pain? & $y / n$ \\
\hline 10 & Has cold decreased the pain? & $y / n$ \\
\hline 11 & Does the affected tooth feel too high? & $y / n$ \\
\hline
\end{tabular}

SIP symptomatic irreversible pulpitis, SAP symptomatic apical periodontitis

the remaining 173 patients diagnosed with either SIP or SAP, the ratio between females to males was 70/103. The average patient age was 40 years and ranged between 18 and 78 years. The ratio between mandibular and maxillary teeth was $107 / 66$. One hundred and thirty of the teeth were molars, 33 premolars and 10 anterior teeth. One hundred and forty-seven of the teeth were multi-rooted, and the remaining 26 were single-rooted. Eleven of the 103 teeth diagnosed with SAP were rootfilled. There were no statistical differences regarding tooth types between SIP and SAP in the current cohort $(P>0.05)$.

\section{Pain levels}

Pain levels were statistically similar $(P>0.05)$ for both conditions under investigation. Median NRS-11 ratings were 8 for both SAP and SIP, with similar interquartile ranges: 2 for SAP, 1 for SIP. There was also no difference $(P>0.05)$ in pain levels between male and female patients. Eighty-one percent $(81 \%)$ of the patients used analgesics within the last $24 \mathrm{~h}$ before seeking emergency treatment. There was no statistical difference between the two conditions under investigation in this regard either.

\section{Key symptoms}

Assessment of the checklist revealed that large differences in the symptomology of SAP and SIP were found for pain duration, pain on cold, and the feeling that the tooth was too high (Table 3). The prediction model, based on the multiple imputed data set, is summarized in Table 4. Furthermore, the estimated odds ratios and $95 \%$ confidence intervals $(\mathrm{CI})$ are displayed. The discriminative ability of the prediction model resulted in an
Table 2 Clinical findings used in the current study to differentiate between SIP and SAP

\begin{tabular}{lll}
\hline Criterion & SIP & SAP \\
\hline Sensitivity to carbon dioxide snow & + & - \\
Radiographically widened ligament space & $+/-$ & + \\
Periapical radiolucency & - & $+/-$ \\
Swelling or sinus tract & - & - \\
\hline
\end{tabular}

SIP symptomatic irreversible pulpitis, SAP symptomatic apical periodontitis, + positive response or sign clearly present,+ - mixed response or not present in all cases, - negative response or clearly absent

Note: sensitivity to percussion was not included, as almost all of the acutely painful teeth in this study responded positive to percussion

AUC of 0.796 (95 \% CI: 0.728-0.864). The decision tree analysis (Fig. 2) resulted in a tree with splits according to pain on cold, awareness of the tooth feeling too high, and pain duration. The first indicator for SAP was a reported absence of pain to cold stimuli. In teeth that did have a history of pain triggered by cold stimuli, the decision tree correctly identified SAP in $72 \%$ of the teeth that felt too high and had hurt for less than one week. The overall sensitivity of the tree was $95 \%$ and the specificity was $31 \%$. The positive predictive value was $67 \%$.

\section{Discussion}

The current study related symptoms to clinical findings. From an immediate treatment planning perspective, SAP is the more critical condition, and its diagnosis should not be missed [16]. It was confirmed that the reported presence or absence of pain to cold stimuli was a first differentiator between the clinical diagnoses of SAP and SIP. Moreover, decision analysis identified additional symptoms associated with a diagnosis of SAP also in teeth with a history of pain to cold stimuli. If these felt too high and had hurt for less than one week, the

Table 3 Descriptive statistics: counts of symptoms in patients diagnosed with SIP $(N=70)$ and SAP $(N=103)$

\begin{tabular}{lllll}
\hline Question & SIP & $\%$ & SAP & $\%$ \\
\hline Sleep disturbed & 56 & $80.0 \%$ & 87 & $84.5 \%$ \\
Pain has decreased & 11 & $15.7 \%$ & 12 & $11.7 \%$ \\
Pain less 1 week & 32 & $45.7 \%$ & 72 & $69.9 \%$ \\
Constant pain & 25 & $35.7 \%$ & 51 & $49.5 \%$ \\
Radiating pain & 25 & $35.7 \%$ & 26 & $25.2 \%$ \\
Sharp pain & 38 & $54.3 \%$ & 37 & $35.9 \%$ \\
Pain on chewing & 49 & $70.0 \%$ & 89 & $86.4 \%$ \\
Pain on hot & 28 & $40.0 \%$ & 29 & $28.2 \%$ \\
Pain on cold & 53 & $75.7 \%$ & 37 & $35.9 \%$ \\
Cold lessens pain $^{\text {Tooth feels high }}{ }^{a}$ & 5 & $7.1 \%$ & 18 & $17.5 \%$ \\
\hline SIP symptomatic irreversibe & 20 & $28.6 \%$ & 50 & $48.5 \%$ \\
\hline
\end{tabular}

SIP symptomatic irreversible pulpitis, SAP symptomatic apical periodontitis ${ }^{\text {aT }}$ This variable had 11 missing values (teeth were bridge abutments) 
Table 4 Results of the prediction model for SAP

\begin{tabular}{lcl}
\hline & Odds ratio & $95 \% \mathrm{Cl}$ \\
\hline Sleep disturbed & 1.0 & $0.4-2.6$ \\
Pain has decreased & 0.9 & $0.3-2.8$ \\
Pain less 1 week & 2.1 & $1-4.4$ \\
Constant pain & 1.6 & $0.8-3.4$ \\
Radiating pain & 0.6 & $0.3-1.5$ \\
Sharp pain & 0.5 & $0.2-1$ \\
Pain on chewing & 2.5 & $1-6.3$ \\
Pain on hot & 0.8 & $0.4-1.6$ \\
Pain on cold & 0.2 & $0.1-0.5$ \\
Cold lessens pain & 0.8 & $0.2-2.9$ \\
Tooth feels high & 1.9 & $0.9-4.3$ \\
\hline
\end{tabular}

$S A P$ symptomatic apical periodontitis, $\mathrm{Cl}$ confidence Interval

probability that SAP was diagnosed was still $72 \%$. A set of questions was thus identified that should be helpful in clinics and for cross-sectional studies to discern between SAP and SIP.

The current study is limited by the fact that data were generated in one single city. The relative frequency of the conditions under investigation is influenced by demographics, the local health care system and socioeconomic factors [23]. It would appear that in older studies, SIP was more frequent than SAP [24], while newer investigations including the current work found the opposite [3]. This could be due to the fact that caries, the main cause for symptomatic pulpitis [9, 25], has steadily declined in industrialized countries [26]. Acute forms of apical periodontitis including abscess formation, on the other hand, can occur as late complications in crowned teeth and/or root-filled counterparts [27].

While current pulp tests and two-dimensional radiology are insufficient to determine the exact histological condition of asymptomatic teeth [28-30], the methods that were used in the present study to differentiate between the two acute conditions under investigation can be regarded as sound. The teeth diagnosed with SIP mostly showed a delayed, yet always more pronounced reaction to the cold test compared to healthy counterparts. It has been shown that painful teeth responding with an delayed, but increased and lingering response to the cold test invariably contain a vital pulp or at least vital aspects of the pulp in the apical root canal $[25,28]$. In multirooted teeth some roots may still contain vital tissue that responds to thermal tests, while in other roots the tissue can be partially necrotic [31]. These vital aspects of the pulp inhibit bacterial infection [32]. Consequently, it is fair to state that acutely painful teeth with positive response to cold test differ from counterparts that test negatively in regard to the level of infection. An intra-operative diagnosis upon entering the pulp space was not performed to differentiate between SIP and SAP.

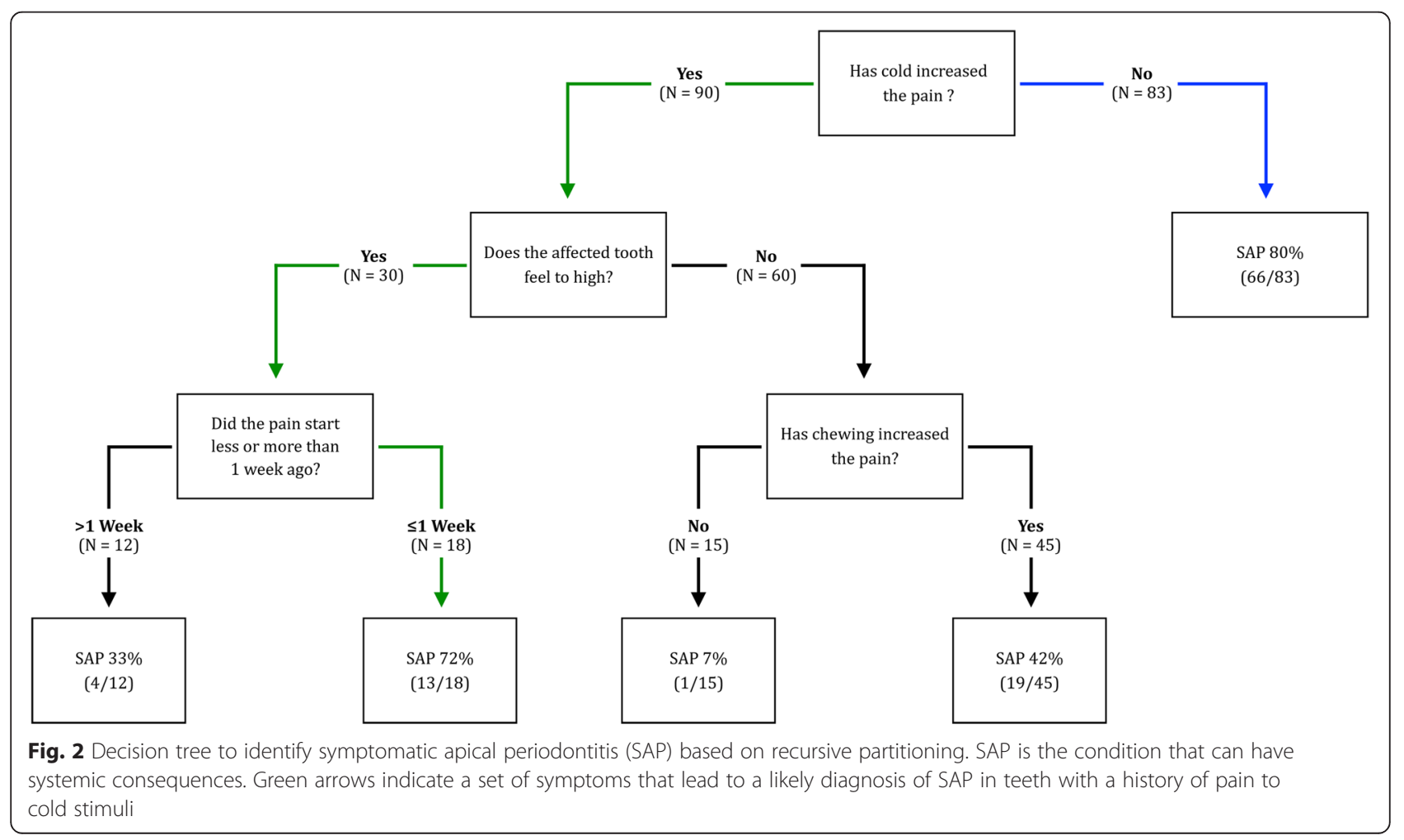


It is known that the intake of analgesics can affect endodontic diagnosis. The pain perception itself can decrease, or the response to clinical test like tooth percussion can be reduced [33]. However, the teeth under investigation were severely painful. Although $81 \%(N=$ 140) of the 173 patients included to this study had consumed analgesics within the past $24 \mathrm{~h}$ before seeking emergency treatment, $79 \%(N=136)$ of them reported severe pain at levels between 7 and 10 on a NRS-11 scale [21]. Moreover, almost all teeth (92\%; $N=159)$ featured a painful response to percussion. In contrast the influence of analgesics on pulpal sensitivity test has shown to be negligible [34]. Consequently, the influence of analgesics on the diagnostic procedures performed here is expected to be low. It needs to be acknowledged that SIP and SAP are clinically defined separate diagnostic entities based on a set of criteria defined by international consensus (and as such reported in textbooks and major journal articles; [6]). SIP, SAP, and later AAA are biological, dynamically evolving stages of the same underlying bacterial infection. The transition from pulpitis or chronic apical periodontitis to acute apical periodontitis is clinically important, because it marks the point when a spread of the infection from the pulp space to the periapical tissues is about to occur [4]. However, the transitions between these conditions are rarely clearcut [32]. Furthermore, transitions from one stage to the next can be fast. This is reflected in the current data in that $35.9 \%$ of the patients diagnosed with acute SAP reported a history of sensitivity to cold (Table 3). Almost half of SAP teeth did not show clear apical radiolucencies typical for chronic apical periodontitis (Table 2). This can also be taken as an indicator that the inflammatory conditions in the periapical tissues developed more rapidly than any radiologically discernible bone changes occurred. This is in line with observations published by other authors [10]. Some recent research suggests that bone changes do occur early in the disease process, when the pulp is still vital [35]. These changes, however, are not necessarily detected on single-tooth radiographs [36]. Cone beam computed tomography (CBCT) was shown to be more sensitive in detecting apical disease [36]. The observation on the dynamics of periapical bone changes made here warrants further investigation and verification using CBCT.

Earlier authors concur with the present results in that pain to cold stimuli [28] and the feeling that the affected tooth is too high [13] can help to differentiate between SIP and SAP. However, it has to be cautioned that these authors did not use the current nomenclature of the diseases under investigation, and thus direct comparisons are limited. The current finding that sensitivity to cold is the main indicator for an inflamed vital pulp corresponds to the observations made in the only study on this topic in humans with induced pulpal inflammation [37]. Consequentially, this should further the support of cold testing e.g., carbon dioxide snow being the main clinical test, in conjunction with radiographic images, to diagnose SIP [25]. The patients diagnosed with SAP reported significantly more often the perception that the pain-causing tooth felt too high compared to patients diagnosed with SIP. This observation may be explained by the spread of the inflammation to the periapical ligament. The accumulation of inflammatory exudate may extrude the affected tooth, thus rendering it tender to occlusion [15]. Other common clinical tests were performed in this study, but these were of little value in differentiating between the acute forms of the conditions under investigation. As an example, 159 (92\%) of the 173 teeth in this study were positive to percussion, with no difference between the two conditions. This is in line with published reports: the percussion test has little to no diagnostic value $[28,38]$. This is especially the case with painful teeth [39]. Nevertheless, the test is used in the current $\mathrm{AAE}$ nomenclature [6]. In accordance with the AAE terminology each tooth that is sensitive to percussion has a periapical diagnosis of "symptomatic apical periodontitis [10, 40]. In the current report, however, the comprehensive diagnosis of the acute forms of SAP before abscess formation versus SIP was based on the thermal responsiveness of the pulp (Table 2).

Numerical rating scales have been validated, and are commonly used for the assessment of pain intensities [7, 41]. The NRS-11 came to use here because of the interview character of this study. In contrast to a visual analogue scale, which is also commonly used in studies on endodontic pain, the NRS can be verbally applied without any visual aids. The 11 potentially differentiating symptoms used in this communication were selected based on the clinical experience of the investigators and common textbook recommendations [20]. They have been assessed, albeit not in the precise formulation attempted here, in various previous studies to differentiate between teeth of different clinical conditions [13, 28]. Pain intensity of endodontically involved teeth causing the patient to seek emergency care was investigated in several previous studies $[2,7,40,42]$. The current mean NRS-11 pain levels between 7 and 8 correspond well to those measured using a VAS scale on emergency patients in previous studies $[7,42]$. The current findings confirm an earlier report in that pain intensity had no differentiating value in the context of acute endodontic conditions [7]. This was not the case for pain duration though. Teeth affected by pulpits apparently hurt for more than one week before the pain reached a level that caused the patient to seek emergency care.

The current approach to facilitate the diagnostic decision between SAP and no SAP (SIP) has been two-fold. 
Initially, a prediction model including all available clinical information was constructed to assess the discriminative ability of the full checklist as measured with the AUC. The resulting AUC was nearly $80 \%$, which is an indication that the full model is in some degree valuable for the distinction between patients with SAP and SIP. However, the use of a prediction model based on 11 variables may be difficult to implement in a clinical setting unless a respective computer algorithm will be made available, e.g., in the form of a software application. For that reason, the decision tree analysis, which facilitates the distinction into the groups of SAP and SIP following a specific order of questions/key symptoms, was added in a second step (Fig. 2). The overall sensitivity to detect SAP based on the decision tree was $95 \%$. However, as is typical for diagnostic decisions with a high sensitivity, the resulting specificity of the tree was lower (31\%). This result demonstrates that the decision tree can be useful for cross-sectional studies. In a clinical setting, the decision tree may be helpful to advise patients during out-of hours calls.

\section{Conclusions}

This study confirmed that in severely painful teeth, the most specific single symptom to differentiate between SIP and SAP was pain to cold stimuli. In addition, however, decision analysis identified a set of key symptoms to diagnose SAP also in teeth with a history of pain to cold stimuli. If these felt too high and had hurt for less than one week, the probability that SAP was diagnosed was still $72 \%$.

\section{Abbreviations}

AAA, acute apical abscess; AAE, American Association of Endodontists; CBCT, cone beam computed tomography; NRS, numeric rating scale; SAP, symptomatic apical periodontitis; SIP, symptomatic irreversible pulpitis

\section{Acknowledgements}

The authors thank Dr. Heather T. Morris for her help with this manuscript.

\section{Funding}

This research was supported by institutional funds of the Department of Preventive Dentistry, Periodontology, and Cariology, University of Zürich Center for Dental Medicine.

\section{Availability of data and materials}

Data supporting the conclusions are included to the article (Tables 3 and 4).

\section{Authors' contributions}

DKR conceived this research, was the main investigator and wrote the manuscript. GB helped collecting the data. UH and JMB performed the statistical analysis. TA revised the manuscript and supervised the study. All authors read and approved the final manuscript.

\section{Competing interests}

The authors declare that they have no competing interests.

\section{Ethical approval and consent to participate}

The study protocol was approved by the ethics committee Zurich, Switzerland (KEK-ZH-Nr. 2012-0450). Participation was voluntary and privacy and confidentiality of all study information was maintained. Written informed consent was obtained from all patients.

\section{Author details}

'Department of Preventive Dentistry, Periodontology and Cariology, Center of Dental Medicine, University of Zürich, Switzerland, Plattenstrasse 11, CH-8032 Zürich, Switzerland. 'Department of Internal Medicine, Horten Center for Patient Oriented Research and Knowledge Transfer, University of Zürich, Zürich, Switzerland.

Received: 3 December 2015 Accepted: 24 May 2016

Published online: 27 May 2016

\section{References}

1. Lipton JA, Ship JA, Larach-Robinson D. Estimated prevalence and distribution of reported orofacial pain in the United States. J Am Dent Assoc. 1993;124:115-21.

2. Sindet-Pedersen S, Petersen JK, Gotzsche PC. Incidence of pain conditions in dental practice in a Danish county. Community Dent Oral Epidemiol. 1985; 13:244-6.

3. Widstrom E, Pietila I, Nilsson B. Diagnosis and treatment of dental emergencies in two Finnish cities. Community Dent Health. 1990;7:173-8.

4. Nair PN. Apical periodontitis: a dynamic encounter between root canal infection and host response. Periodontol 2000. 1997;13:121-48.

5. Julius D, Basbaum Al. Molecular mechanisms of nociception. Nature. 2001; 413:203-10

6. American Association of Endodontists. AAE consensus conference recommended diagnostic terminology. J Endod. 2009;35:1634.

7. McCarthy PJ, McClanahan S, Hodges J, Bowles WR. Frequency of localization of the painful tooth by patients presenting for an endodontic emergency. J Endod. 2010;36:801-5.

8. Michaelson PL, Holland GR. Is pulpitis painful? Int Endod J. 2002;35:829-32

9. Estrela C, Guedes OA, Silva JA, Leles CR, Estrela CR, Pecora JD. Diagnostic and clinical factors associated with pulpal and periapical pain. Braz Dent J. 2011;22:306-11.

10. Zero DT, Zandona AF, Vail MM, Spolnik KJ. Dental caries and pulpal disease Dent Clin North Am. 2011;55:29-46.

11. Miller J, Elwood PC, Swallow JN. Dental pain. An incidence study. Br Dent J. 1975;139:327-8.

12. Pau A, Croucher R, Marcenes W, Leung T. Development and validation of a dental pain-screening questionnaire. Pain. 2005;119:75-81.

13. Klausen B, Helbo M, Dabelsteen E. A differential diagnostic approach to the symptomatology of acute dental pain. Oral Surg Oral Med Oral Pathol. 1985; 59:297-301.

14. Iqbal M, Kim S, Yoon F. An investigation into differential diagnosis of pulp and periapical pain: a PennEndo database study. J Endod. 2007;33:548-51.

15. Carrotte P. Endodontics: Part 3. Treatment of endodontic emergencies. $\mathrm{Br}$ Dent J. 2004;197:299-305.

16. Nalliah RP, Allareddy V, Elangovan S, Karimbux N, Lee MK, Gajendrareddy P, et al. Hospital emergency department visits attributed to pulpal and periapical disease in the United States in 2006. J Endod. 2011:37:6-9.

17. Siqueira Jr JF, Rocas IN. Microbiology and treatment of acute apical abscesses. Clin Microbiol Rev. 2013:26:255-73.

18. Levine M. Understanding how a dental infection may spread to the brain: case report. J Can Dent Assoc. 2013;79:d9.

19. Asgary S, Eghbal MJ. The effect of pulpotomy using a calcium-enriched mixture cement versus one-visit root canal therapy on postoperative pain relief in irreversible pulpitis: a randomized clinical trial. Odontology. 2010;98:126-33.

20. Hasselgren G, Green D, Scrivani SJ. Tooth related pain. In: Mehta N, Maloney GE, Bana DS, Scrivani J, editors. Head, Face, and Neck Pain Science, Evaluation, and Management: An Interdisciplinary Approach. Hoboken: Wiley-Blackwell; 2010. p. 330-42.

21. Warren Grant Magnusson Clinical Center. Pain intensity instruments. 2003. http://www.mvltca.net/presentations/mvltca.pdf. Accessed 26 May 2016.

22. R Development Core Team. R: A language and environment for statistical computing. In: R Foundation for Statistical Computing V. Vienna: R Foundation for Statistical Computing; 2011. 
23. Pau AK, Croucher R, Marcenes W. Prevalence estimates and associated factors for dental pain: a review. Oral Health Prev Dent. 2003;1:209-20.

24. Hasler JF, Mitchell DF. Analysis of 1628 cases of odontalgia. A corraborative study. J Indianapolis Dent Soc. 1963;17:23-5.

25. Ricucci D, Loghin S, Siqueira Jr JF. Correlation between clinical and histologic pulp diagnoses. J Endod. 2014;40:1932-9.

26. Menghini G, Steiner M, Thomet E, Rath C, Marthaler T, Imfeld T. Further caries decline in Swiss recruits from 1996 to 2006. Schweiz Monatsschr Zahnmed. 2010;120:590-600.

27. Bergenholtz G, Nyman S. Endodontic complications following periodontal and prosthetic treatment of patients with advanced periodontal disease. J Periodontol. 1984;55:63-8.

28. Dummer PM, Hicks R, Huws D. Clinical signs and symptoms in pulp disease. Int Endod J. 1980;13:27-35.

29. Bender IB, Seltzer S. Roentgenographic and direct observation of experimental lesions in bone: II. 1961. J Endod. 2003;29:707-12.

30. Mejare IA, Axelsson S, Davidson T, Frisk F, Hakeberg M, Kvist T, et al. Diagnosis of the condition of the dental pulp: a systematic review. Int Endod J. 2012;45:597-613.

31. Peters DD, Baumgartner JC, Lorton L. Adult pulpal diagnosis. I. Evaluation of the positive and negative responses to cold and electrical pulp tests. J Endod. 1994:20:506-11.

32. Langeland K. Tissue response to dental caries. Endod Dent Traumatol. 1987; 3:149-71.

33. Read JK, McClanahan SB, Khan AA, Lunos S, Bowles WR. Effect of Ibuprofen on masking endodontic diagnosis. J Endod. 2014:40:1058-62.

34. Carnes PL, Cook B, Eleazer PD, Scheetz JP. Change in pain threshold by meperidine, naproxen sodium, and acetaminophen as determined by electric pulp testing. Anesth Prog. 1998;45:139-42.

35. Rechenberg DK, Bostanci N, Zehnder M, Belibasakis GN. Periapical fluid RANKL and IL-8 are differentially regulated in pulpitis and apical periodontitis. Cytokine. 2014;69:116-9.

36. Abella F, Patel S, Duran-Sindreu F, Mercade M, Bueno R, Roig M. Evaluating the periapical status of teeth with irreversible pulpitis by using cone-beam computed tomography scanning and periapical radiographs. J Endod. 2012; 38:1588-91.

37. Lundy T, Stanley HR. Correlation of pulpal histopathology and clinical symptoms in human teeth subjected to experimental irritation. Oral Surg Oral Med Oral Pathol. 1969;27:187-201.

38. Hasler JE, Mitchell DF. Painless pulpitis. J Am Dent Assoc. 1970;81:671-7.

39. Tyldesley WR, Mumford JM. Dental pain and the histological condition of the pulp. Dent Pract Dent Rec. 1970;20:333-6.

40. Owatz CB, Khan AA, Schindler WG, Schwartz SA, Keiser K, Hargreaves KM The incidence of mechanical allodynia in patients with irreversible pulpitis. Endod. 2007;33:552-6.

41. Ferreira-Valente MA, Pais-Ribeiro $J$, Jensen MP. Validity of four pain intensity rating scales. Pain. 2011;152:2399-404.

42. Runyon MS, Brennan MT, Batts JJ, Glaser TE, Fox PC, Norton HJ, et al. Efficacy of penicillin for dental pain without overt infection. Acad Emerg Med. 2004;11:1268-71.

\section{Submit your next manuscript to BioMed Central and we will help you at every step:}

- We accept pre-submission inquiries

- Our selector tool helps you to find the most relevant journal

- We provide round the clock customer support

- Convenient online submission

- Thorough peer review

- Inclusion in PubMed and all major indexing services

- Maximum visibility for your research

Submit your manuscript at www.biomedcentral.com/submit 